\title{
Fracción exhalada de óxido nítrico. Recomendaciones clínicas y procedimiento
}

\section{Fraction of exhaled nitric oxide. Clinical recommendations and procedure}

\author{
Gabriela Cantú-González, ${ }^{*}$ Fabiola Fernández-Figueroa, ${ }^{*}$ Laura Gochicoa-Rangel, ${ }^{*}$ José Luis Miguel-Reyes, ${ }^{*}$ \\ Claudia Vargas-Domínguez, ${ }^{*}$ Roberto Mejía-Alfaro, ${ }^{*}$ Luis Torre-Bouscoulet*
}

\author{
*Departamento de Fisiología Respiratoria, \\ Instituto Nacional de Enfermedades Respiratorias Ismael Cosío Villegas. Ciudad de México.
}

\begin{abstract}
RESUMEN. La medición de la fracción espirada de óxido nítrico, ha permitido cuantificar indirectamente, de forma rápida y confiable la magnitud de la inflamación de la vía aérea en ciertas enfermedades respiratorias, en especial en pacientes con asma. La sencillez de esta técnica la ha convertido en una herramienta útil en el diagnóstico, control y evaluación del cumplimiento terapéutico en pacientes con asma, entre otros padecimientos. Además, mediante la medición de la fracción espirada de óxido nítrico se pueden prevenir agudizaciones debido a que permite detectar y tratar de forma inmediata los incrementos de la actividad inflamatoria. El propósito de este documento es revisar aspectos prácticos de la medición de la fracción espirada de óxido nítrico utilizando un dispositivo portátil. De igual forma, se desea proveer al clínico de herramientas útiles para la apropiada interpretación de los resultados. Consideramos que la medición de la fracción espirada de óxido nítrico es una prueba subutilizada en la práctica neumológica, y es nuestro interés poner a disposición del lector información resumida y útil.
\end{abstract}

Palabras clave: Óxido nítrico, fracción exhalada de óxido nítrico.

\section{Abreviaturas:}

ATS: American Thoracic Society (Sociedad Americana del Tórax)

CEI: Corticoesteroides inhalados

EPOC: Enfermedad pulmonar obstructiva crónica

ERS: European Respiratory Society (Sociedad Respiratoria Europea)

FeON: Fracción exhalada de óxido nítrico

FRDE: Factor de relajación dependiente del endotelio

GMPc: Guanosín monofosfato cíclico

NANC: No-adrenérgico, no-colinérgico

NANCe: No-adrenérgico y no-colinérgico función excitatoria

NANCi: No-adrenérgico y no-colinérgico función inhibitoria

$\mathrm{NO}_{2}$ : Dióxido de nitrógeno

$\mathrm{NOS}_{2}$ : Óxido nítrico sintasa

\section{Correspondencia:}

\section{Dra. Fabiola Fernández-Figueroa}

Departamento de Fisiología Respiratoria.

Instituto Nacional de Enfermedades Respiratorias Ismael Cosío Villegas.

Calzada de Tlalpan 4502,

Colonia Sección XVI. 14080 Ciudad de México.

Tel: +52 55 54871700, ext: 5160

Correo electrónico: nancyfafe@hotmail.com

Trabajo recibido: 18-VII-2012; aceptado: 04-XII-2012.
ABSTRACT. Measuring the fraction of exhaled nitric oxide makes it possible to quantify «in an indirect, quick and reliable» fashion, the magnitude of airway inflammation in patients with respiratory diseases; especially in cases of asthma. Because of its simplicity, this technique has also become a useful tool for diagnosing, monitoring and evaluating the therapeutic compliance in patients with asthma, among other conditions. In addition, measuring fraction of exhaled nitric oxide can help prevent exacerbations because it allows increases in inflammatory activity to be detected and treated immediately. The purpose of this document is to review practical aspects of the fraction of exhaled nitric oxide-measuring technique using a portable device, and to provide clinical physicians with useful instruments that will aid in achieving correct interpretations of results. We consider the fraction of exhaled nitric oxide-measuring technique to be an underutilized test in current respiratory medicine practice, so our motivation is to make practical, summarized information available to readers.

Keywords: Nitric oxide, exhaled fraction of nitric oxide.

ON: Óxído nitríco

ONSe: Isoforma de óxido nítrico sintasa endotelial

ONSi: Isoforma de óxido nítrico sintasa inducible calcio-independiente

ONSn: Isoforma de óxido nítrico sintasa neuronal

PFR: Pruebas de función respiratoria

ppb: Partes por billón

\section{INTRODUCCIÓN}

El óxido nítrico $(\mathrm{ON})$ es una molécula simple, considerada hasta fines de los años 80 únicamente como un producto derivado de la combustión gaseosa. En 1987 gracias a los estudios de Moncada e Ignarro en cultivos de células endoteliales sometidas a estímulos oxidativos, el ON fue reconocido como el equivalente al factor de relajación dependiente del endotelio, debido a la similitud del comportamiento farmacológico de ambos compuestos. ${ }^{1,2}$

La posibilidad de medir el ON se ha desarrollado notablemente en los últimos 15 años, midiéndose de forma indirecta por sus derivados biológicos o directamente por 
quimioluminiscencia. El ON tiene un papel elemental en la regulación de múltiples sistemas biológicos; se han publicado más de 70,000 artículos que relacionan a esta molécula con funciones fisiológicas fundamentales, como la relajación vascular, la agregación plaquetaria, la neurotransmisión o la oxidación mitocondrial..$^{3-5}$

El ON se sintetiza en las células endoteliales y epiteliales de la vía aérea, así como en algunas células inflamatorias de la mucosa respiratoria tales como: los mastocitos, neutrófilos, células cebadas y los macrófagos; de ahí el ON se difunde en forma gaseosa hacia la luz de la vía aérea. ${ }^{1,5}$ La síntesis se produce tras la acción de una enzima sintasa sobre el aminoácido L-arginina, formándose L-citrulina y $\mathrm{ON}$ como un radical libre altamente inestable que reacciona con derivados lipídicos, nitrogenados o acuosos de las estructuras celulares cercanas (Figura 1).

La concentración de $\mathrm{ON}$ en aire exhalado resulta, por tanto, del balance entre la producción de ON y su capacidad de reacción con el medio que le rodea. También interfieren otros factores como la capacidad de difusión a través de la mucosa bronquial, el grado de ventilación alveolar y el flujo de aire. ${ }^{6}$ Se han identificado tres isoformas de óxido nítrico sintasa $\left(\mathrm{NOS}_{2}\right)$, dos de ellas constitutivas calcio-dependientes: la endotelial (eONS) o tipo III, la neural (nONS) o tipo I y una forma inducible calcio-independiente (iONS) o tipo II. $^{5}$

\section{FUNCIONES FISIOLÓGICAS DEL ON}

En el sistema respiratorio el ON actúa como un broncodilatador débil, relajando la musculatura lisa bronquial, por medio de la activación de la guanilato ciclasa y aumentando el guanosín monofosfato cíclico (GMPc).

La broncodilatación es mayor en la vía aérea traqueobronquial que en la vía aérea de pequeño calibre. También el ON está implicado en la ruta metabólica del «tiol» para formar «nitrosotioles», los cuales están presentes en la vías respiratorias de sujetos sanos; éstos tienen una vida media sustancialmente mayor que el ON, y poseen potente actividad broncodilatadora que es independiente de la vía de la GMPc. Los nitrosotioles están presentes en concentraciones suficientes para influir en el tono de las vías respiratorias. ${ }^{7}$

Además de los sistemas clásicos colinérgicos y adrenérgicos que impulsan el tono motor bronquial, existe el sistema nervioso no-adrenérgico y no-colinérgico (NANC) en las vías respiratorias de los animales y los seres humanos. Funcionan mediando la contracción (función excitatoria NANCe) o controlando la relajación (función inhibitoria NANCi).

Los estudios realizados en los últimos años demuestran que el ON actúa como un neurotransmisor de la función inhibitoria (NANCi); y en el hombre la respuesta inhi-

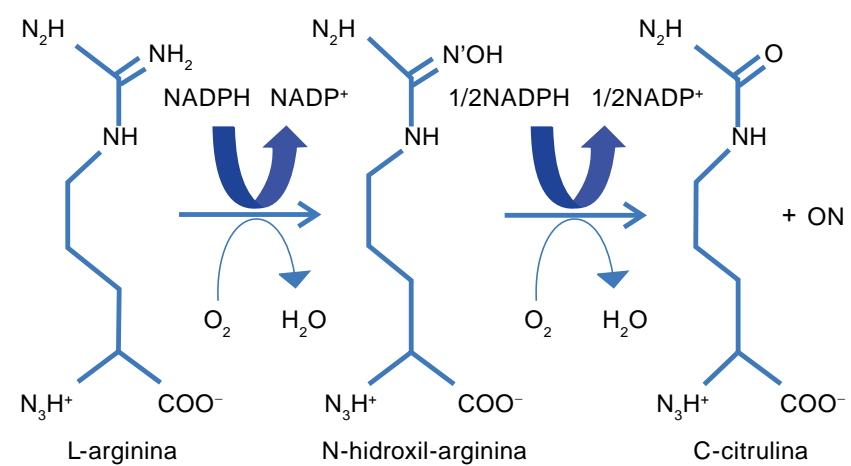

Figura 1: Reacción de la ON sintasa que produce óxido nítrico y citrulina a partir de arginina.

bitoria (NANCi) de la vía aérea central y periférica está completamente mediada por el ON. ${ }^{7}$

El ON tiene propiedades antimicrobianas; el aumento de la producción de ON se asocia a la resistencia del huésped, mientras que el bloqueo de su síntesis aumenta la replicación del agente infeccioso en los macrófagos infectados. También parece tener particular importancia en los sistemas de defensa contra patógenos intracelulares, y puede actuar causando daño mediante la desaminación del ADN e inhibiendo enzimas. ${ }^{7}$ El ON funciona, además, como modulador de la diferenciación celular y amplificador de la inflamación en la vía aérea. ${ }^{6}$ Las altas concentraciones locales de ON favorecen la inhibición de la diferenciación de las células CD4 + T helper (Th) en Th1, las cuales son productoras de interleucina-2 e interferón- $\gamma$; del mismo modo, el ON favorece el aumento de células Th2 que secretan interleucina 4, 5 y 10 , favoreciendo la producción de inmunoglobulina $\mathrm{E}$, así como la adherencia y acumulación de eosinófilos. ${ }^{7}$ EI ON favorece el aumento del edema, del exudado plasmático y del despegamiento del epitelio bronquial. ${ }^{8}$

El ON desempeña un papel clave en prácticamente todos los aspectos biológicos del pulmón, y ha sido implicado en la fisiopatología de enfermedades pulmonares como el asma; los pacientes asmáticos cursan con elevadas concentraciones de $\mathrm{ON}$ exhalado en su respiración. ${ }^{9-11}$

El propósito de este documento es revisar aspectos prácticos de la medición de la FeON utilizando un dispositivo portátil, así como proveer al clínico de herramientas útiles para la apropiada interpretación de los resultados. Consideramos que la medición de la FeON es una prueba subutilizada en la práctica neumológica, y es nuestro interés poner a disposición del lector información resumida y útil. No intentamos agotar el tema, para obtener información detallada acerca de la FeON; se recomienda al lector revisar los documentos publicados por la Sociedad Americana del Tórax (ATS, por sus siglas en inglés). 


\section{Fracción espirada de óxido nítrico (FeON). Definición}

La FeON es una medida cuantitativa del ON proveniente de la vía aérea. El ON es un mediador gaseoso producido endógenamente por la sintasa de $\mathrm{ON}$, y es comúnmente considerado como un marcador indirecto de inflamación de la vía aérea. Se ha demostrado que las personas con asma, fibrosis quística, enfermedad pulmonar obstructiva crónica (EPOC), entre otras, exhalan elevadas concentraciones de ON que disminuyen en respuesta a los corticosteroides.

La medición de la FeON es una prueba no invasiva, es fácil de realizar en niños y en pacientes con obstrucción grave del flujo aéreo, y no tiene riesgo alguno para el paciente. ${ }^{12,13}$

\section{Metodología para la medición}

El ON se mide con métodos espectroscópicos que incluyen quimioluminiscencia, espectroscopia visible ultravioleta y resonancia paramagnética de electrones. El más utilizado es la quimioluminiscencia que se basa en la medición de la intensidad de la radiación fluorescente emitida después de la oxidación química de ON por el ozono. Esta reacción fotoquímica emite una luz en el rango del infrarrojo que es detectada por un tubo fotomultiplicador con una respuesta lineal. El producto de esta reacción es el dióxido de nitrógeno $\left(\mathrm{NO}_{2}\right)$, y emite un fotón. El número total de fotones producidos es proporcional a la concentración de ON. ${ }^{7}$

En el mercado existen varios modelos de equipos para la medición por quimioluminiscencia, como SIR N-6008 ${ }^{\circledR}$; LR 2000 analyser ${ }^{\circledR}$; NIOX Aerocrine ${ }^{\circledR}$; todos estos equipos comparten los fundamentos necesarios para la realización de la técnica.

Los métodos de medición electroquímicos se basan en la oxidación electroquímica del $\mathrm{ON}$ detectado por electrodos sólidos. Si la corriente generada durante esta reacción de oxidación es linealmente proporcional a la concentración, la corriente de oxidación puede utilizarse como una señal analítica y medirse en amperes o volts. ${ }^{14}$ Un ejemplo de este tipo es un equipo portátil de gran utilidad en el contexto clínico de los últimos años, y que funciona con un sensor electroquímico marca NIOX-MINO (Aerocrine, Solna, Suecia); este equipo es de pequeñas dimensiones ( $24 \mathrm{x}$ $13 \times 10 \mathrm{~cm}$ ) y tiene $800 \mathrm{gr}$ de peso, permite la medición de FeON domiciliario y por el propio paciente debido a su sencillez en el uso. El dispositivo tiene un sensor lumínico y acústico para facilitar y asegurar el flujo espiratorio, el resultado aparece de forma digital en una pantalla visible por el paciente, los resultados se guardan mediante una tarjeta digital lo que facilita el seguimiento de las mediciones (Figura 2). Las recomendaciones y consideraciones técnicas que se hacen en este documento son únicamente tomando en cuenta dicho equipo portátil. Para aspectos técnicos de otros dispositivos se recomienda revisar las especificaciones del fabricante. ${ }^{15}$

La medición de FeON se expresa en partes por billón (ppb). Existen dos formas de medición de la FeON. La primera es llamada «medición en línea» (on-line) y se refiere a la exhalación directa en el aparato de medición. La segunda es llamada «medición fuera de línea» (off-line) que se refiere a la exhalación en una bolsa impermeable para recolección de gases, los cuales posteriormente se «muestrean» para la medición de ON. Esta última, puede utilizarse para la recolección en sitios lejanos al analizador (que puede incluir salas de hospital, clínicas, escuelas o laboratorios a distancia). Cualquiera de las dos formas de medición es válida y precisa; sin embargo, no son intercambiables debido a diferencias en la metodología, principalmente la relacionada con la velocidad del flujo.

La velocidad de la exhalación afecta a la FeON; los flujos rápidos dan lecturas bajas de FeON; en diferencia con flujos lentos la, FeON puede estar elevada. Se recomienda una velocidad de flujo de $0.05 \mathrm{~L} / \mathrm{seg}$ para las mediciones en línea y de $0.35 \mathrm{~L} / \mathrm{seg}$ en las mediciones fuera de línea; en ambos se considera una exhalación de por lo menos tres segundos para conseguir una meseta. ${ }^{15}$

\section{Repetibilidad de la medición}

El coeficiente de variación de la medición de la FeON en individuos sanos es de aproximadamente 10\% (alrededor

Figura 2:

Analizador electroquímico portátil de óxido nítrico.

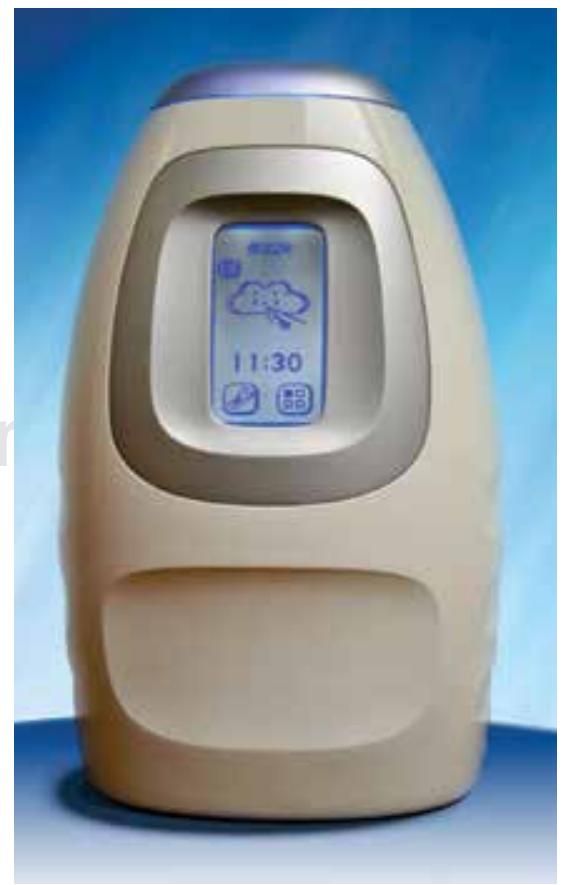


de $4 \mathrm{ppb}$ ), mientras que la variación en los individuos con asma oscila entre el 20 y el $40 \%{ }^{7-9}$ Así, puesto que un cambio de $20 \%$ puede ser debido a la variación en la medición, las guías ATS/ERS recomiendan un cambio de al menos $20 \%$ para indicar un aumento o disminución significativos en la FeON. ${ }^{16}$

Aunque hay muy pocos estudios que aclaren lo que constituye un cambio clínicamente significativo en pacientes individuales, se recomienda efectuar la medición de la FeON antes de realizar otras pruebas de función respiratoria (PFR), debido a que se incrementa la tasa de falsos negativos. ${ }^{15}$

\section{Consideraciones demográficas}

Los niveles de FeON son generalmente menores en niños que en adultos. En promedio, los menores de 12 años tienen aproximadamente $5 \mathrm{ppb}$ menos que en adultos. El valor de FeON en hombres es aproximadamente 25\% superior a lo observado en mujeres. El índice de masa corporal se asoció inversamente con el FeON en personas con asma, y la pérdida excesiva de peso también puede afectar la FeON. ${ }^{17}$

\section{Indicaciones para la medición de la FeON}

Las indicaciones para la medición de la FeON de acuerdo a las guías de la ATS 2011 son las siguientes:

1. Para establecer el diagnóstico de inflamación eosinofílica de la vía aérea.

2. Para «predecir» la respuesta al tratamiento con esteroides inhalados en individuos con síntomas crónicos sugestivos de inflamación de la vía aérea.

a) Para evaluar la respuesta potencial o falta de respuesta a los antiinflamatorios, sobre todo los corticosteroides inhalados (CSI) y guiar los cambios en las dosis.

b) En asma crónica persistente para establecer una línea de base durante la estabilidad clínica y el seguimiento posterior.

c) Para ayudar en la evaluación de la adherencia a los medicamentos antiinflamatorios.

3. Para ayudar al diagnóstico de asma en situaciones que se requiera de mayor evidencia objetiva.

4. Para monitorizar la inflamación de la vía aérea en pacientes con diagnóstico de asma.

a) Para evaluar si la inflamación de las vías está contribuyendo al mal control del asma, en particular en la presencia de comorbilidades (ej. rinosinusitis, reflujo gastroesofágico, obesidad o exposición a alérgenos).
A los pacientes que se les realiza seguimiento con mediciones seriadas de $\mathrm{FeON}$, se les puede ajustar (usualmente disminuir hasta un $40 \%$ ) la dosis de CSI. ${ }^{18}$

5. Resulta útil en el diagnóstico diferencial de los niños preescolares con sibilancias recurrentes, edad donde la espirometría forzada y el esputo inducido son más difíciles de realizar.

\section{ASOCIACIONES}

Debido a que hay varios fenotipos de asma la determinación del subtipo puede ayudar al médico a decidir qué tipo de tratamiento emplear. Dada la relación entre la inflamación eosinofílica y la capacidad de respuesta de esteroides la medición de FeON sugiere su uso como indicador indirecto, no sólo de la inflamación eosinofílica sino de la potencial capacidad de respuesta de esteroides.

Es cierto que hay poca evidencia que demuestre que la inflamación eosinofílica de las vías respiratorias aumenta directamente el FeON; la inflamación eosinofílica puede afectar la medición de FeON de manera indirecta a través de $\mathrm{NOS}_{2}$ o a través de otras vías enzimáticas. En función de la presencia de inflamación eosinofílica en las vías aéreas, las mediciones de FeON en una población específica puede ser útil para identificar pacientes con síntomas similares al asma que son susceptibles de beneficiarse o no del tratamiento con corticosteroides. ${ }^{18,19}$

\section{Situaciones en las que FeON podría ser útil}

Las guías internacionales ayudan para la interpretación de FeON y dan un panorama para su utilidad clínica. La medición seriada de FeON es un método viable y confiable para supervisar la inflamación de la vía aérea en pacientes asmáticos. ${ }^{20}$ La presencia de síntomas respiratorios compatibles, limitación al flujo aéreo y un FeON elevado tienen una alta sensibilidad y especificidad para diagnosticar asma; ${ }^{21-23}$ sin embargo, no existe asociación con la gravedad ${ }^{20} \mathrm{ni}$ con el FEV1. ${ }^{21,22,24}$

Una de las mayores utilidades del FeON es su capacidad para predecir la respuesta a la terapia oral con corticosteroides y esteroides inhalados en pacientes con asma, EPOC y en pacientes con tos crónica. En los pacientes con asma, la FeON disminuye rápidamente al iniciar el uso de corticosteroides y antileucotrienos, pero no con nedocromil ni teofilina. No se ha demostrado asociación entre FeON y calidad de vida en pacientes asmáticos. ${ }^{16}$

El papel exacto de las mediciones de FeON en pacientes con EPOC aún está por definirse; existen estudios con un número significativo de pacientes que han demostrado que la respuesta a los corticosteroides es mayor en pacientes con EPOC y eosinofília en el esputo. Esto plantea la posibilidad de que FeON pueda ser utilizado para predecir la 
capacidad de respuesta a los esteroides en el tratamiento de EPOC o durante las exacerbaciones.

La medición de FeON no es exclusiva para pacientes con asma, también puede incrementarse en pacientes con atopia, rinitis alérgica y bronquitis eosinofílica sin asma. ${ }^{25}$ Se ha documentado que la FeON correlaciona significativamente con el grado de hiperrespuesta bronquial, reversibilidad al broncodilatador y atopia. ${ }^{23}$

Existe asociación entre el grado de inflamación bronquial medida por FeON y eosinofília en sangre, eosinófilos en el esputo, eosinofília en mucosas y eosinofília en lavado bronquioloalveolar. ${ }^{26,27}$

La asociación entre FeON e hiperrespuesta bronquial fue débil en pacientes con larga evolución del asma, probablemente debido a la remodelación de la vía aérea. Esta teoría se aborda en un estudio reciente donde fue demostrado una mayor correlación entre la FeON e hiperrespuesta bronquial a la metacolina en niños (10-17 años) asmáticos comparado contra lo observado en el grupo de adultos (18-35 años). ${ }^{13}$

\section{Contraindicaciones}

- No se recomienda realizarla en pacientes que presenten condiciones que favorezcan la elevación de valores de $\mathrm{FeON}$, aun en presencia de síntomas sugestivos de asma (Tablas 1 y 2).

- Sujetos con déficit cognitivo y que no puedan realizar la maniobra.

\section{PROCEDIMIENTO PARA MEDICIÓN DE NO EXHALADO CON EL ANALIZADOR ELECTROQUÍMICO PORTÁTIL NIOX-MINO}

\section{Equipo y materiales}

1.1 Analizador electroquímico portátil NIOX-MINO (Aerocrine, Solna, Suecia) (Figura 2).

1.2 Sensor para NIOX-MINO.

1.3 Rejilla para ON NIOX-MINO.

1.4 Filtro desechable marca NIOX (Figura 3).

1.5 El analizador electroquímico puede ser conectado a una PC mediante un cable USB de forma opcional, a fin de que el paciente pueda visualizar el incentivo.

\section{Control de calidad del equipo}

2.1 El control de calidad para este equipo consiste de dos partes. La primera es un control positivo que podría ser cualquier individuo sano pulmonar que debe estar clínicamente libre de atopías. Éste debe realizar tres mediciones, una por día durante tres días consecutivos. El aparato genera un valor promedio de estas tres mediciones, las cuales deben estar entre 5 y 40 ppb. Después (día cuatro) se realiza una cuarta medición, la cual debe de estar en $\pm 10 \mathrm{ppb}$ del valor promedio para que el control de calidad sea aprobado. Estas cuatro mediciones deben realizarse en un plazo no mayor de siete días. ${ }^{15}$

La segunda parte, es un control negativo que consiste en una muestra de gas libre de ON, generada del aire

Tabla 1: Condiciones clínicas que comúnmente elevan la FeON. 2,18

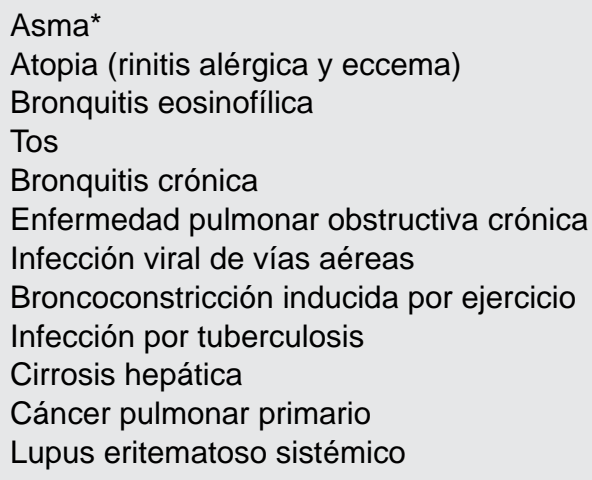

* Demostrado que FeON tiene aplicación clínica.

Tabla 2: Condiciones que pueden producir cambios en las concentraciones de FeON.,18

\begin{tabular}{|l|l|}
\hline \multicolumn{1}{|c|}{ Lo aumentan } & \multicolumn{1}{c|}{ Lo disminuyen } \\
\hline Cafeína & Alcohol \\
Fibrosis quística & Altitud \\
Dieta rica en nitratos & Insuficiencia cardíaca \\
Sarcoidosis pulmonar & Ejercicio 30 min antes \\
Tabaquismo activo & Obesidad \\
Tabaquismo pasivo & Hipertensión pulmonar \\
Cese de tabaquismo & Espirometría \\
Esclerosis sistémica & Síndrome de discinesia \\
& ciliar primaria \\
& Hemorragia alveolar \\
& Infección VIH \\
\hline
\end{tabular}

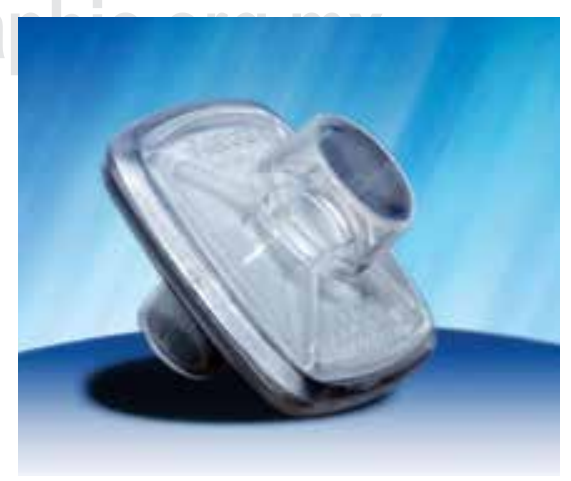

Figura 3:

Filtro desechable para el Niox-Mino. 
ambiente ( $<5 \mathrm{ppb}$ ); este procedimiento se realiza todos los días automáticamente por el equipo al encenderse. ${ }^{15}$

2.2 Una vez realizado este procedimiento, el aparato está listo para su uso clínico, el cual debe ser realizado en un período de entre siete y veinte días. El aparato señala con un asterisco cuándo es necesario volver a realizar el procedimiento. ${ }^{15}$

2.3 Para el control biológico el fabricante recomienda un mínimo de dos individuos que, como ya se mencionó, puedan ser seleccionados del personal del laboratorio de función pulmonar, siempre y cuando estén sanos de sus pulmones, libres de atopias, no fumadores, mayores de 18 años de edad. Este es el control positivo, y se sabe porque se le asigna un número para cada control biológico (del uno al cuatro), para que la realización sucesiva de estos controles biológicos siempre sea con el número previamente asignado. ${ }^{15}$

2.4 Las mediciones para control de calidad no tienen impacto en el número de pruebas contenidas en el sensor. $^{15}$

\section{Preparación del paciente}

3.1 Se debe de evitar el tabaquismo, consumo de alimentos ricos en nitratos como la lechuga, bebidas con cafeína y agua por lo menos dos horas antes de la prueba; asegurarse de que el paciente no haya hecho ejercicio 30 min antes de la prueba y no haber ingerido alcohol 12 horas antes de realizar la medición de FeON. ${ }^{14}$

3.2 Se recomienda realizar la maniobra para medición de FeON previamente a otras PFR, ya que las maniobras de espiración repetidas disminuyen los valores. ${ }^{14}$

3.3 No se recomienda la suspensión de medicamentos de uso rutinario; sin embargo, debe de realizarse un registro de ellos debido a que los esteroides inhalados y los modificadores de leucotrienos disminuyen los valores de FeON, mientras que la L-arginina los aumenta. ${ }^{14,17}$

3.4 El ritmo circadiano tiene efecto sobre la FeON; por la mañana los valores suelen ser menores que por la tarde. Por ello, se recomienda que las mediciones en un mismo individuo sean realizadas en horas similares del día. ${ }^{18}$

\section{Procedimiento previo al examen}

4.1 El técnico se presenta con el paciente.

4.2 Conecte el equipo a la fuente de luz. Puede conectarlo a una PC que tenga instalado el programa con el incentivo incluido con el analizador, y verifique que no requiera de realizar el control de calidad.

4.3 Se debe revisar que los datos de la solicitud del estudio correspondan al paciente. Anotar el diagnóstico, la indicación de la prueba y si es de seguimiento o de primera vez, además se le asigna un número de identificación. Para este fin se puede utilizar el software incluido con el equipo para registro de datos o de acuerdo a los lineamientos de cada laboratorio para el manejo de datos.

4.4 Explique al paciente la maniobra y el uso del filtro.

\section{Instrucciones para el paciente y realización de la prueba}

5.1 Solicite al paciente que adopte la posición sedente con la espalda recta y la vista en la pantalla de la computadora, para que pueda observar el incentivo (en caso de que tenga esta opción).

5.2 Solicite al paciente que vacíe totalmente los pulmones realizando una maniobra espiratoria hasta alcanzar volumen residual.

5.3 Solicite al paciente que sostenga con sus dos manos el analizador portátil a la altura de su boca, y que se coloque la boquilla del filtro.

5.4 Pida al paciente realizar una inspiración profunda dentro del filtro hasta lograr capacidad pulmonar total. En caso de contar con el incentivo visual, el técnico debe estar familiarizado con éste.

5.5 Pida al paciente realizar una exhalación suave hasta que la alerta lumínica del analizador permanezca constante, al igual que la alerta auditiva. En caso de contar con el incentivo visual el técnico debe estar familiarizado con éste; esto indica que se está realizando una exhalación con un flujo de $50 \mathrm{~mL} / \mathrm{s}$ con una presión de entre 5 y $20 \mathrm{cmH}_{2} \mathrm{O}$. El tiempo de duración de la exhalación es de al menos seis segundos para mayores de 12 años y cuatro segundos para los menores de 12 años.

5.6 Solicite al paciente retirarse de la boquilla.

5.7 Retire el filtro y coloque en su lugar el tapón.

5.8 Espere aproximadamente un minuto a que aparezca el resultado.

5.9 La pantalla del analizador muestra el resultado en ppb, así como el número de identificación y el número de la prueba en forma consecutiva (por lo cual ninguna prueba puede tener el mismo número que otra). ${ }^{15}$

\section{Informe de los resultados}

6.1 La ATS en la estandarización para la medición de ON exhalado del 2005 recomienda la realización de dos pruebas con una variabilidad menor al $10 \%$ entre cada una e informar el promedio de ambas pruebas como resultado, p.ej., si la primera medición es de $23 \mathrm{ppb}$ y la segunda de $25 \mathrm{ppb}$ hay una variabilidad de $8.2 \%$, y el promedio a reportarse es de 24 ppb; 


\section{REFERENCIAS}

1. de Diego DA. Nitric oxide in asthma: what good does it do?. Arch Bronconeumol 2010;46:157-159.

2. Nathan C, Xie QW. Nitric oxide synthases: roles, tolls, and controls. Cell 1994;78:915-918.

3. Ignarro LJ, Buga GM, Wood KS, Byrns RE, Chaudhuri G. Endothelium derived relaxing factor produced and released from artery and vein is nitric oxide. Proc Natl Acad Sci USA 1987;84:9265-9269.

4. Palmer RM, Ashton DS, Moncada S. Vascular endothelial cells synthesize nitric oxide from L-arginine. Nature 1988;333:664-666.

5. Cobos BN, Pérez YEG, Sardón PO, Reverté BC, Garther S, Korta MJ. Exhaled nitric oxide in children: a noninvasive marker of airway inflammation. Arch Bronconeumol 2008:44:41-51.

6. Lane C, Knight D, Burgess S, et al. Epithelial inducible nitric oxide synthase activity is the major determinant of nitric oxide concentration in exhaled breath. Thorax 2004;59:757-760.

7. Ricciardolo FL. Multiple roles of nitric oxide in the airways. Thorax 2003;58:175-182.

8. Ekroos H, Karjalainen J, Sarna S, Laitinen LA, Sovijarvi AR. Shortterm variability of exhaled nitric oxide in young male patients with mild asthma and in healthy subjects. Respir Med 2002;96:895-900.

9. Latzin P, Beck J, Griese M. Exhaled nitric oxide in healthy children: variability and a lack of correlation with atopy. Pediatr Allergy Immunol 2002;13:37-46.

10. Pijnenburg MW, Floor SE, Hop WC, De Jongste JC. Daily ambulatory exhaled nitric oxide measurements in asthma. Pediatr Allergy Immunol 2006;17:189-193.

11. Reid DW, Johns DP, Feltis B, Ward C, Walters EH. Exhaled nitric oxide continues to reflect airway hyperresponsiveness and disease activity in inhaled corticosteroid-treated adult asthmatic patients. Respirology 2003:8:479-486.

12. Ozkan M, Dweik RA. Nitric oxide and airway reactivity. Clin Pulm Med 2001;8:199-206.

13. Dweik RA, Sorkness RL, Wenzel S, et al. Use of exhaled nitric oxide measurement to identify a reactive, at-risk phenotype among patients with asthma. Am J Respir Crit Care Med 2010;181:1033-1041.

14. ATS/ERS. Recommendations for standardized procedures for the online and offline measurement of exhaled lower respiratory nitric oxide and nasal nitric oxide. Am J Respir Crit Care Med 2005;171:912-930 doi:10.1164.

15. Procedure for NIOX MINO and quality control procedure user manual EPM-000073, version 04, May 2008.
16. Lim KG, Mottram C. The use of fraction of exhaled nitric oxide in pulmonary practice. Chest 2008;133:1232-1242.

17. ATS/ERS. Recommendations for standardized procedures for the on-line and off-line measurement of exhaled lower respiratory nitric oxide and nasal nitric oxide in adults and children-1999. This official statement of the American Thoracic Society was adopted by the ATS Board of Directors, July 1999. Am J Respir Crit Care Med 1999;160:2104-2117.

18. Dweik RA, Boggs PB, Erzurum SC, et al. An official ATS clinical practice guideline: interpretation of exhaled nitric oxide levels (FENO) for clinical applications. Am J Resp Crit Care Med 2011;184:602-615.

19. Zietkowski Z, Bodzenta-Lukaszyk A, Tornasiak MM, Skiepko R, Szmitkowski M. Comparison of exhaled nitric oxide measurement with conventional tests in steroid-naive asthma patients. J Investig Allergol Clin Immunol 2006;16:239-246.

20. Smith AD, Cowan JO, Filsell S, et al. Diagnosing asthma: comparisons between exhaled nitric oxide measurements and conventional tests. Am J Respir Crit Care Med 2004;169:473-478.

21. Shaw DE, Berry MA, Thomas M, et al. The use of exhaled nitric oxide to guide asthma management: a randomized controlled trial. Am J Respir Crit Care Med 2007;176:231-237.

22. Bodini A, Peroni D, Loiacono A, et al. Exhaled nitric oxide daily evaluation is effective in monitoring exposure to relevant allergens in asthmatic children. Chest 2007;132:1520-1525.

23. Ludviksdottir D, Diamant Z, Alving K, Bjermer L, Malinovschi A. Clinical aspects of using exhaled $\mathrm{NO}$ in asthma diagnosis and management. Clin Respir J 2012;6:193-207.

24. Moore WC, Bleecker ER, Curran-Everett D, et al. Characterization of the severe asthma phenotype by the National Heart, Lung, and Blood Institute's Severe Asthma Research Program. J Allergy Clin Immunol 2007; 119:405-413.

25. Smith O. Nobel Prize for NO research. Nat Med 1998:4:1215.

26. Dupont LJ, Demedts MG, Verleden GM. Prospective evaluation of the validity of exhaled nitric oxide for the diagnosis of asthma. Chest 2003; 123:751-756.

27. Smith AD, Cowan JO, Brassett KP, Herbison GP, Taylor DR. Use of exhaled nitric oxide measurements to guide treatment in chronic asthma. N Engl J Med 2005;352:2163-2173.

28. Sociedad Española de Neumología y Cirugía Torácica (SEPAR). Sociedad Española de Alergología e Inmunología Clínica. Guía Española para el Manejo del Asma. 2009. www.gemasma.com

Los autores declaran no tener conflicto de intereses. 